\title{
Neonatal Necrotizing Fasciitis after Circumcision: A Case Report and Review of Literature
}

\author{
Ada L. Rivera Cruz¹, Zasha F. Vázquez Colón¹, Hector Quintero², Cesar Carballo Cuello², \\ Victor N. Ortiz Justiniano ${ }^{3,4^{*}}$ \\ ${ }^{1}$ Puerto Rico Children's Hospital, Bayamón, Puerto Rico \\ ${ }^{2}$ Universidad Central del Caribe School of Medicine, Bayamón, Puerto Rico \\ ${ }^{3}$ Pediatric Surgery Department, Puerto Rico Children's Hospital, Bayamón, Puerto Rico \\ ${ }^{4}$ UPR School of Medicine, San Juan, Puerto Rico \\ Email: "paponoel2@gmail.com
}

Received 7 December 2015; accepted 5 March 2016; published 8 March 2016

Copyright (C) 2016 by authors and Scientific Research Publishing Inc.

This work is licensed under the Creative Commons Attribution International License (CC BY).

http://creativecommons.org/licenses/by/4.0/

c) (i) Open Access

\begin{abstract}
Background: Necrotizing fasciitis is a soft tissue infection that occurs predominantly in adults. Although rare, only 70 cases have been reported in neonates. This entity, most commonly caused by multiple organisms is often fatal, with $50 \%$ mortality. The combination of a low incidence and high mortality in this subgroup strengthens the need for early diagnosis, prompt recognition and immediate surgical treatment in order to improve survival. Aim: The aim of this case report is to bring awareness of a clinical condition that relies mostly on physical examination and where early diagnosis and immediate surgical treatment are needed in order to improve survival. Case presentation: We report the case of an 8-day-old boy presenting neonatal necrotizing fasciitis after circumcision. Patient presented with lower back erythema and areas of fluctuation. He was started on antibiotic therapy and 24 hours after admission, he was taken to the operating room for multiple fasciotomies, debridement and cleansing of affected areas. Findings were consistent with necrotizing fasciitis. After surgical intervention, local care was given until complete healing was achieved, approximately one month after admission. Conclusion: The surgeon must be attentive for the possible occurrence of this lethal infection that may develop post operatively after clean or clean-contaminated procedures. Preparation for treating these patients immediately with adequate fluid resuscitation, appropriate antibiotics and aggressive surgical debridement of non-viable tissue will provide the maximal potential for recovery. These prompt measures will offer the best chance for survival and eventually decrease the $50 \%$ mortality rate associated to the neonatal population.
\end{abstract}

${ }^{*}$ Corresponding author.

How to cite this paper: Cruz, A.L.R., Colón, Z.F.V., Quintero, H., Cuello, C.C. and Justiniano, V.N.O. (2016) Neonatal Necrotizing Fasciitis after Circumcision: A Case Report and Review of Literature. Open Journal of Pediatrics, 6, 29-33.

http://dx.doi.org/10.4236/ojped.2016.61006 
Keywords

Cellulitis, Abscess, Subcutaneous, Erythema, Penrose, Debridement, Granulation

\section{Introduction}

Necrotizing Fasciitis (NF) is a rare and life threatening subcutaneous tissue infection. In the neonatal population, it occurs most commonly as a complication of surgical procedures such as circumcisions, or infections such as omphalitis and balanitis [1] [2]. NF is characterized by a widespread fascial necrosis with separation and infarction of the overlying skin and subcutaneous tissue [3]. The affected area may be erythematous, swollen, warm, shiny and painful [4]. Infants and neonates who develop this disease are characteristically healthy, unlike the adult population in which diabetes and immunosuppression serve as predisposing risk factors [5]. In adults, most cases arise in the extremities, while in neonates lesions predominantly occur in the abdominal wall and trunk area, predisposing for a mortality of nearly 50\% [6]. The combination of a low incidence and high mortality in this subgroup strengthens the need for early diagnosis, prompt recognition and immediate surgical treatment in order to improve survival [7]. We present the case of an 8-day-old infant who developed necrotizing fasciitis after a circumcision.

\section{Case Report}

Case of an 8-day-old baby boy had undergone circumcision at two days of age and was doing well until one day prior to admission when he developed fever. He was taken to the Emergency Room at another institution and on physical examination was found with lower back erythema consistent with cellulitis. He was transferred to our institution due to suspicion of abscess formation and for pediatric surgery evaluation. On physical examination he was found irritable, afebrile, with some areas of mild fluctuation and induration in the left buttock. A lumbosacral and buttock sonogram was performed and results came negative for abscess. Laboratory values revealed leukopenia at $3 \times 10^{9} / \mathrm{L}(3000$ cells $/ \mu \mathrm{L})$ and a significantly elevated C-Reactive Protein (CRP) at $25 \mathrm{mg} / \mathrm{dl}$ (238.1 nmol/L). Broad-spectrum antibiotic therapy was started with Vancomycin and Gentamicin, along with intravenous fluid (IVF) hydration and bowel rest. After 24 hours of admission he was found irritable, with unchanged cellulitis and persistent lower back fluctuation. He was taken to the operating room for multiple fasciotomies, debridement and cleansing of affected areas. On gross examination there was grayish fascia with scattered necrosis and multiple areas of abscesses, consistent with necrotizing fasciitis and abscesses. Penrose drains were left in place to keep the wounds open (Figure 1). Daily bedside debridement was performed and on postoperative day five, he was taken to the operating room for Penrose drains removal. Wound cultures were positive for Staphylococcus aureus and Citrobacter freundi susceptible to Vancomycin and Meropenem; antibiotics were tailored accordingly. Local care was performed on a daily basis with hydrogel wound dressing and subsequently with antimicrobial silver dressing until an adequate wound bed of granulation tissue was present.

Epithelialization and contraction were achieved by use of topical silver nitrate. Complete healing was reached approximately one month after admission (Figure 2). The patient was discharged home and followed up closely in the outpatient clinics.

\section{Discussion}

In 1871, a confederate army surgeon, Joseph Jones was credited for describing NF infections as hospital gangrene [8]. Wilson was the first to describe this condition as necrotizing fasciitis in 1952. He stated the typical clinical presentation of NF that is tachycardia, fever and local infection leading to necrosis of the subcutaneous and superficial tissues [9]. This pathology most commonly occurs in adults, mainly affecting the extremities, contrary to neonates where it is considered rare and mostly involves the abdomen, low back and perineal area. In the neonatal population, it is usually associated with one of several underlying conditions such as omphalitis, mastitis, balanitis, necrotizing enterolocolitis, immunodeficiency, bullous impetigo, surgical wound infection, or trauma [10]-[14]. Our patient presented with NF a few days after circumcision, an unusual finding scarcely reported in the literature. 


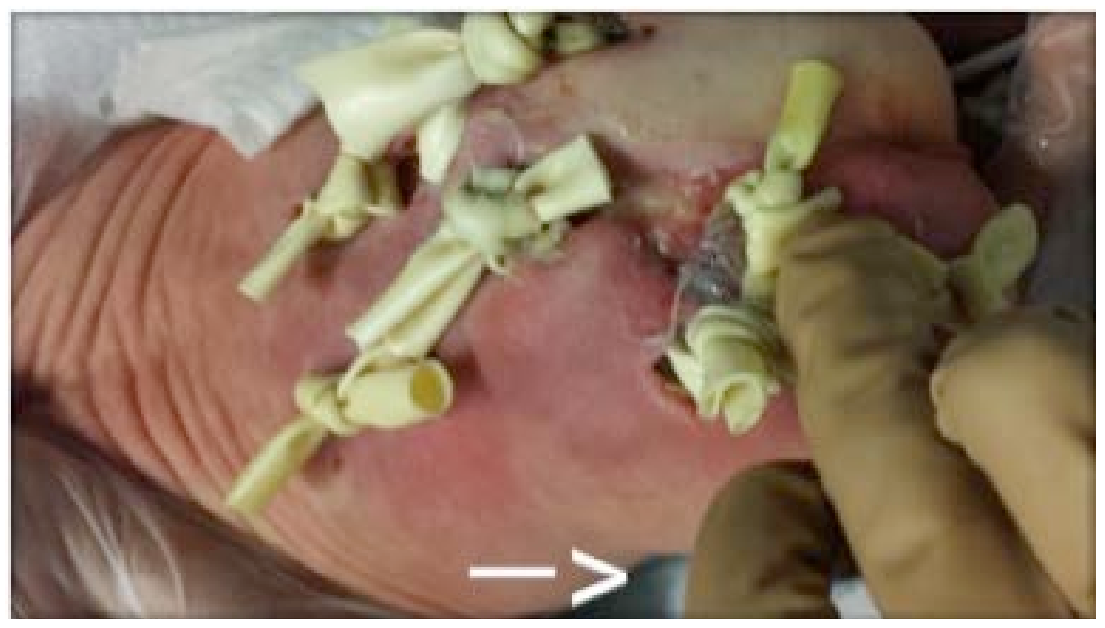

Figure 1. Extensive erythema evident after debridement and placement of Penrose drains along with sloughed off skin and some devitalized tissue. Arrow direction is caudalad.

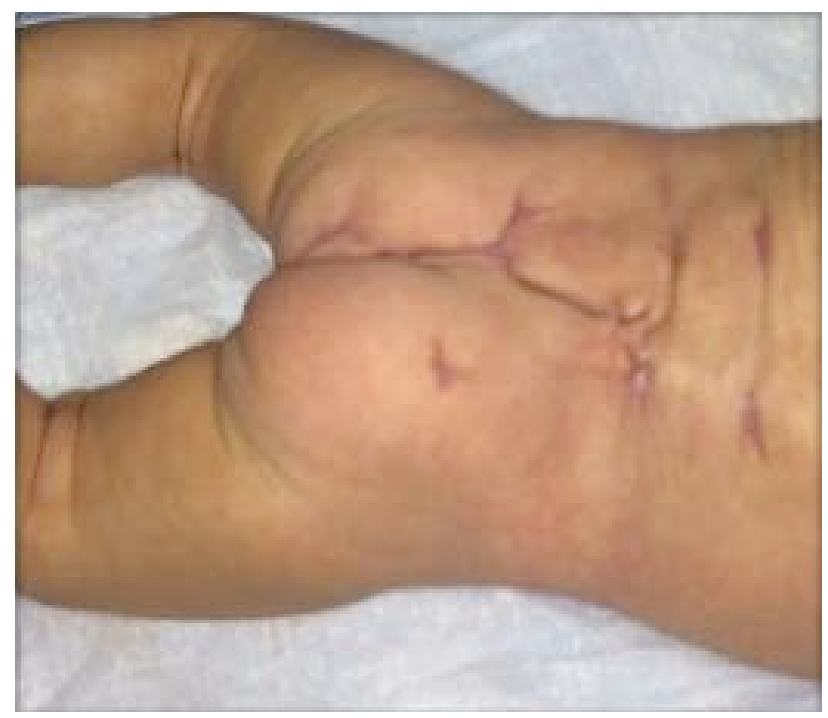

Figure 2. Complete wound healing approximately 3 months after admission.

An estimated one in three males worldwide is circumcised for religious, cultural or medical reasons [15]. In a large meta-analysis of prospective and retrospective series, Weiss et al. reported the median frequency of any adverse event of 1.5\% [16]. Most commonly, adverse events include bleeding and infection. In rare cases, urethrocutaneous fistula, meatal stenosis, scabs, glanular amputation or necrosis, and necrotizing fasciitis, among others, have been documented.

Necrotizing soft tissue infections occurs when bacteria infect the superficial fascia. It can be caused by two bacteriological entities, type 1 which is polymicrobial and type 2 by group A beta hemolytic Streptococcal species. Type 1 is a mixed infection caused by aerobic and anaerobic bacteria and is highly correlated to risk factors such as diabetes, vascular disease, immune compromise, and recent surgery, such as circumcision in neonates. Type 2 occurs primarily among healthy individuals with recent skin injury, laceration or surgery [17]. There are limited cases reported of NF in the neonatal population, most are type 1 (polymicrobial) or type 2 with group A Streptococcus in combination with Staphylococcus aureus. Wound cultures of our patient were positive for Staphylococcus aureus and Citrobacter species, thus it can be categorized as type 1 .

This pathology is thought to occur because bacteria secrete pyogenic exotoxins that stimulate inflammatory cytokine production by the host, producing a cascade of cytodestructive events such as shock, tissue destruction and possible organ failure [16]. Microvascular thrombosis leads to fat and fascial necrosis, causing a greater 
systemic inflammatory response that may lead to disfiguring wounds or potential multiorgan failure [18].

Early in the disease, skin changes are variable as well as laboratory findings, for which timely diagnosis is based on a high index of suspicion. Clinical clues for diagnosis are: pain out of proportion to the clinical appearance of the affected skin, marked soft tissue edema, rapid symptom progression, and signs of systemic toxicity. Diagnosis of NF is mainly clinical, supported by advanced symptoms and sometimes crepitus.

Although laboratory studies are an important aspect in the workup for NF, the diagnosis is established definitively by surgical exploration. Radiographic studies, such as soft tissue plain films, or computed tomography (CT) scans, can be useful to assess muscle involvement, but it is not reliable for evaluation of the fascia. It is important to emphasize that surgical intervention should not be delayed while waiting for laboratories or other diagnostic studies. None of these diagnostic studies have shown to be effective in the diagnosis of NF. It relies mostly on high index of clinical suspicion, as we have previously stated [18].

The gold-standard management of NF is surgical. It should consist of extensive fascial and soft tissue debridement along with hemodynamic support and antibiotic therapy. In our case, patient showed significant improvement after surgical debridement was done and treatment with intravenous antibiotics was started. Early intervention upon his worsening course allowed for his positive outcome.

\section{Conclusion}

The surgeon must be attentive for the possible occurrence of this lethal infection that may develop post operatively after clean or clean-contaminated procedures. Preparation for treating these patients immediately with adequate fluid resuscitation, appropriate antibiotics and aggressive surgical debridement of non-viable tissue will provide the maximal potential for recovery. These prompt measures will offer the best chance for survival and eventually decrease the $50 \%$ mortality rate associated to the neonatal population [19].

\section{References}

[1] Hsieh, W., Yang, P., Chao, H. and Lai, J. (1999) Neonatal Necrotizing Fasciitis: A Report of Three Cases and Review of the Literature. Pediatrics, 103, e53-e53. http://dx.doi.org/10.1542/peds.103.4.e53

[2] Jamal, N. and Teach, S. (2011) Necrotizing Fasciitis. Pediatric Emergency Care, 27, 1195-1199. http://dx.doi.org/10.1097/PEC.0b013e31823b583c

[3] Murphy, J., Granger, R., Blair, G., Miller, G., Fraser, G. and Magee, J. (1995) Necrotizing Fasciitis in Childhood. Journal of Pediatric Surgery, 30, 1131-1134. http://dx.doi.org/10.1016/0022-3468(95)90004-7

[4] Sudarsky, L., Laschinger, J., Coppa, G. and Spencer, F. (1987) Improved Results from a Standardized Approach in Treating Patients with Necrotizing Fasciitis. Annals of Surgery, 208, 661-665. http://dx.doi.org/10.1097/00000658-198711000-00018

[5] Lally, K., Atkinson, J., Woolley, M. and Mahour, G. (1984) Necrotizing Fasciitis. Annals of Surgery, 199, $101-103$. http://dx.doi.org/10.1097/00000658-198401000-00017

[6] Sawin, R., Schaller, R., Tapper, D., Morgan, A. and Cahill, J. (1994) Early Recognition of Neonatal Abdominal Wall Necrotizing Fasciitis. The American Journal of Surgery, 167, 481-484. http://dx.doi.org/10.1016/0002-9610(94)90239-9

[7] Bliss, D., Healey, P. and Waldhausen, J. (1998) Necrotizing Fasciitis after Plastibell Circumcision. The Journal of Urology, 159, 1408. http://dx.doi.org/10.1016/S0022-5347(01)63646-2

[8] Marcus, J., Hussong, J., Gonzalez, C. and Dumanian, G. (1998) Risk Factors in Necrotizing Fasciitis: A Case Involving Cryptococcus Neoformans. Annals of Plastic Surgery, 40, 80-83. http://dx.doi.org/10.1097/00000637-199801000-00018

[9] Wilson, B. (1952) Necrotizing Fasciitis. The American Surgeon, 18, 416-431.

[10] Kosloske, A., Cushing, A., Borden, T., Woodside, J., Klein, M., Kulasinghe, H., et al. (1981) Cellulitis and Necrotizing Fasciitis of the Abdominal Wall in Pediatric Patients. Journal of Pediatric Surgery, 16, 246-251. http://dx.doi.org/10.1016/S0022-3468(81)80673-2

[11] Sawin, R., Schaller, R., Tapper, D., Morgan, A. and Cahill, J. (1994) Early Recognition of Neonatal Abdominal Wall Necrotizing Fasciitis. The American Journal of Surgery, 167, 481-484. http://dx.doi.org/10.1016/0002-9610(94)90239-9

[12] Farrell, L., Karel, S., Davis, P., Bellinger, M. and Ballantine, T. (1988) Postoperative Necrotizing Fasciitis in Children. Pediatrics, 82, 874-879.

[13] Wilson, H. (1973) Acute Necrotizing Fasciitis in Childhood. American Journal of Diseases of Children, $125,591$. 
http://dx.doi.org/10.1001/archpedi.1973.04160040087018

[14] Brook, I. (1998) Microbiology of Necrotizing Fasciitis Associated with Omphalitis in the Newborn Infant. Journal of Perinatology, 18, 28-30.

[15] World Health Organization and Joint United Nations Programme on HIV/AIDS. (2008) Male Circumcision: Global Trends and Determinants of Prevalence, Safety and Acceptability. WHO Press, Geneva.

[16] Weiss, H., Larke, N., Halperin, D. and Schenker, I. (2010) Complications of Circumcision in Male Neonates, Infants and Children: A Systematic Review. BMC Urology, 10, 2. http://dx.doi.org/10.1186/1471-2490-10-2

[17] Hasham, S., Matteucci, P., Stanley, P. and Hart, N. (2005) Necrotising Fasciitis. BMJ, 330, 830-833. http://dx.doi.org/10.1136/bmj.330.7495.830

[18] Stevens, D., Bisno, A., Chambers, H., Dellinger, E., Goldstein, E., Gorbach, S., et al. (2014) Practice Guidelines for the Diagnosis and Management of Skin and Soft Tissue Infections: 2014 Update by the Infectious Diseases Society of America. Clinical Infectious Diseases, 59, e10-e52. http://dx.doi.org/10.1093/cid/ciu296

[19] Abbott, R., Marcus, J., Few, J., Farkas, A. and Jona, J. (1999) Necrotizing Fasciitis in Infancy: An Uncommon Setting and a Prognostic Disadvantage. Journal of Pediatric Surgery, 34, 1432-1434. http://dx.doi.org/10.1016/S0022-3468(99)90031-3

\section{Abbreviations}

Necrotizing fasciitis (NF), C-reactive protein (CRP), intravenous fluids (IVF), computed tomography (CT) 